\title{
A one-period memory folk theorem for multilateral bargaining games
}

Citation for published version (APA):

Herings, P. J. J., Meshalkin, A. V., \& Predtetchinski, A. (2017). A one-period memory folk theorem for multilateral bargaining games. Games and Economic Behavior, 103(May 2017), 185-198.

https://doi.org/10.1016/j.geb.2015.11.006

Document status and date:

Published: 01/05/2017

DOI:

10.1016/j.geb.2015.11.006

Document Version:

Publisher's PDF, also known as Version of record

Document license:

Taverne

Please check the document version of this publication:

- A submitted manuscript is the version of the article upon submission and before peer-review. There can be important differences between the submitted version and the official published version of record.

People interested in the research are advised to contact the author for the final version of the publication, or visit the DOI to the publisher's website.

- The final author version and the galley proof are versions of the publication after peer review.

- The final published version features the final layout of the paper including the volume, issue and page numbers.

Link to publication

\footnotetext{
General rights rights.

- You may freely distribute the URL identifying the publication in the public portal. please follow below link for the End User Agreement:

www.umlib.nl/taverne-license

Take down policy

If you believe that this document breaches copyright please contact us at:

repository@maastrichtuniversity.nl

providing details and we will investigate your claim.
}

Copyright and moral rights for the publications made accessible in the public portal are retained by the authors and/or other copyright owners and it is a condition of accessing publications that users recognise and abide by the legal requirements associated with these

- Users may download and print one copy of any publication from the public portal for the purpose of private study or research.

- You may not further distribute the material or use it for any profit-making activity or commercial gain

If the publication is distributed under the terms of Article $25 \mathrm{fa}$ of the Dutch Copyright Act, indicated by the "Taverne" license above, 


\title{
A one-period memory folk theorem for multilateral bargaining games
}

\author{
P. Jean-Jacques Herings*, Andrey Meshalkin, Arkadi Predtetchinski \\ Department of Economics, Maastricht University, Netherlands
}

\section{A R T I C L E I N F O}

\section{Article history:}

Received 8 November 2013

Available online 7 December 2015

\section{JEL classification:}

$\mathrm{C} 72$

$\mathrm{C} 78$

Keywords:

Dynamic games

Bargaining

Folk theorem

Subgame perfect equilibrium

One-period recall

\begin{abstract}
A B S T R A C T
We study strategies with one-period recall in the context of a general class of multilateral bargaining games. A strategy has one-period recall if actions in a particular period are only conditioned on information in the previous and the current period. We show that if players are sufficiently patient, given any proposal in the space of possible agreements, there exists a subgame perfect equilibrium such that the given proposal is made and unanimously accepted in period zero. As a corollary we derive that also perpetual delay can be sustained as a subgame perfect equilibrium. Our strategies are pure and have one-period recall, and we do not make use of a public randomization device. The players' discount factors are allowed to be heterogeneous. We also construct a finite automata representation of our strategy profile.
\end{abstract}

(c) 2015 Elsevier Inc. All rights reserved.

\section{Introduction}

One of the most important problems in economic theory is the bargaining problem. The bargaining problem studies how agents make an agreement when they can achieve a particular set of feasible payoffs by collaborating. A bargaining game consists of a sequence of proposals and responses to the proposals. If a proposal is accepted by all the players, the game ends. If a proposal is rejected by at least one player, the game continues and the next proposal is made.

We provide a folk theorem for a general class of multilateral bargaining games. The main result of the paper is that any feasible payoff vector can be sustained as a subgame perfect equilibrium outcome using strategies with one-period recall, provided that the players are sufficiently patient. A strategy profile is said to have one-period recall if the players' actions in any given round of bargaining may only be conditioned on actions in the previous and the current rounds. The constructed strategy profile is pure and we do not rely on a public randomization device to establish our folk theorem. As a corollary we derive that also perpetual delay can be sustained as a subgame perfect equilibrium outcome using strategies with one-period recall. We allow the players to have heterogeneous discount factors.

Folk theorems constitute a class of theorems which state that any individually rational outcome can be sustained as an equilibrium. Early contributions to the folk theorem literature are by Friedman (1971) and by Rubinstein (1979). Fudenberg and Maskin (1986) have proved a folk theorem in repeated games with discounting, where subgame perfect equilibrium is used as the solution concept. Since bargaining games do not belong to the class of repeated games, they are not covered by these results.

\footnotetext{
* Corresponding author.

E-mail addresses: P.Herings@maastrichtuniversity.nl (P.J.J. Herings), A.Rybakov@maastrichtuniversity.nl (A. Meshalkin), A.Predtetchinski@maastrichtuniversity.nl (A. Predtetchinski).
} 
Fudenberg and Yamamoto (2011) prove a folk theorem for stochastic games generalizing an earlier result by Dutta (1995). A crucial assumption in both contributions is that of irreducibility: starting from any given state, any other state is visited with a positive probability, irrespective of the moves of any particular player. In bargaining games, some of the states are terminal, and the irreducibility condition is clearly violated. Moreover, both Dutta (1995) and Fudenberg and Yamamoto (2011) assume the set of states and the set of actions to be finite, whereas a large part of the bargaining literature studies infinite action sets. The existing folk theorems for repeated games and for stochastic games therefore do not cover the bargaining model.

One of the main results in the field of bargaining has been proved in Rubinstein (1982). Rubinstein (1982) studies two-person alternating offers bargaining and shows that there is a unique subgame perfect equilibrium in this model. In the unique subgame perfect equilibrium, the proposal of the first proposer is immediately accepted by his opponent. The folk theorem does evidently not hold for two-player bargaining games. We therefore study bargaining games with at least three players in this paper.

The proof of Rubinstein (1982) does not work for bargaining problems with more than two players. As reported in Binmore et al. (1992), one of the first extensions to the three-person case was made by Shaked. In Shaked's example, Player 1 starts by making a proposal which describes each player's share of a unit surplus. The other players must accept or reject this proposal sequentially. If the proposal is accepted by all players, it is implemented and the game ends. If the proposal is rejected by one of the players, the next period begins and Player 2 makes a new proposal. Negotiation continues in this way. It is shown that any efficient payoff vector can be supported by a subgame perfect equilibrium if the common discount factor is sufficiently high.

Herrero (1985) obtains a result similar to Shaked for the case with three or more players, though we will explain that the construction used in Herrero (1985) is not complete. Haller (1986) also considers the case with three or more players in a game where players vote simultaneously on a proposal. Haller (1986) shows that any efficient division of a unit surplus can be supported as a subgame perfect equilibrium irrespective of the value of the discount factor. While these papers have identified that the driving force for the multiplicity of equilibrium payoffs is that a responder can be compensated by rejecting a deviating offer, the extent of such multiplicity of equilibrium payoffs in a general setting and the key factors that drive such multiplicity are less clear. It is here that our paper contributes.

All constructions used in the literature so far rely on strategies which require infinite recall for all players. The action of a player in a given time-period depends on the whole history of play. In particular, the strategy of every player at any given time-period depends on the actual play in period zero. Infinite recall allows for the punishment of a player, who has deviated from his strategy only once, during the whole remainder of the game. Several authors have questioned the plausibility of such behavior.

Aumann (1981) discusses some of the options to narrow down the definition of equilibrium to avoid unreasonable predictions and mentions bounded recall as a way of modeling bounded rationality in repeated games. Sabourian (1998) characterizes the set of bounded recall pure subgame perfect equilibria in a repeated game setting without discounting. His results indicate that the equilibrium set expends fast in the length of recall. Cole and Kocherlakota (2005) show in a repeated game context with imperfect public monitoring that for some parameter settings the assumption of bounded recall may reduce the set of equilibrium payoffs to a singleton. To obtain such a result, however, they also make strong symmetry assumptions with respect to the strategies under consideration. Bhaskar et al. (2013) study subgame perfect equilibria in stochastic games that are purifiable and have bounded recall. Equilibrium strategies are purifiable if they also constitute an equilibrium of a perturbed game with independent private payoff perturbations in the sense of Harsanyi (1973). They show that only Markovian equilibria have bounded recall and are purifiable. Barlo et al. (2009) prove that the folk theorem in repeated games continues to hold even if one restricts attention to strategies with one-period recall.

We consider a general specification of the multilateral bargaining model and explore the existence of subgame perfect equilibria under the strong bounded recall restriction that players' actions may only be conditional on actions in the previous and the current period. Our bargaining protocol covers many existing models as special cases. At the beginning of each round of bargaining, nature chooses the proposer and the order of responses as summarized by a permutation of the set of players. We do not place any restrictions on the moves by nature. These moves could be random and depend on the entire history of play. In particular, the bargaining protocol is allowed to have infinite recall. We make only weak assumptions on the set of feasible payoffs and allow for sets that are non-convex or discrete.

Special cases of our bargaining protocol with alternating or rotating proposers are described in Rubinstein (1982) and Herrero (1985). We cover protocols with time-invariant recognition probabilities as studied in Binmore (1987) and Banks and Duggan (2000). Models where the proposer is selected by means of an underlying Markov process generalize these approaches, see Merlo and Wilson (1995), Kalandrakis (2006), and Herings and Predtetchinski (2010), and are also special cases of our bargaining protocol. Part of the literature studies endogenous bargaining protocols, where a player who rejects becomes the next proposer, a bargaining protocol introduced in Selten (1981) and also used in Chatterjee et al. (1993) in coalitional bargaining theory. Our set-up allows for endogenous protocols as well, where not only rejections affect the choice of the next proposer, but also the contents of previous proposals may influence this choice, something which is not covered in the bargaining literature so far.

We allow for heterogeneous discount factors. Unlike the classical folk theorem in Fudenberg and Maskin (1986), where players have identical discount factors, Lehrer and Pauzner (1999) find that in two-player repeated games with heterogeneous discount factors, not all feasible individually rational payoffs can be supported by an equilibrium, even when both 
players become very patient. They also point out that it is difficult to characterize the feasible payoff set in an $n$-player repeated game. Chen (2008) shows the possibility of a player obtaining a subgame perfect equilibrium payoff below his effective minimax value in a three-player repeated game. As our main result demonstrates, no such difficulties arise for multilateral bargaining games with heterogeneous discount factors. We show that any feasible payoff vector can be sustained as a subgame perfect equilibrium outcome using strategies with one-period recall, provided that the players' discount factors exceed a certain threshold. The threshold is independent of the payoff vector in question. It is completely characterized by the set of feasible payoffs.

The rest of the paper is organized as follows. In Section 2 we discuss our class of multilateral bargaining games and we describe how existing models fit into it. In Section 3 we analyze the results of Herrero (1985). We show that these results rely on the use of strategies with infinite recall. In Section 4 we present a motivating example, which illustrates how a payoff vector can be supported as a subgame perfect equilibrium in one-period recall strategies. In Section 5 we prove the main result, the folk theorem for a general class of multilateral bargaining games. We also construct subgame perfect equilibrium strategies with one-period recall that lead to perpetual disagreement. In Section 6 we describe the strategies constructed for the proof of the folk theorem by means of a finite automaton. Section 7 concludes.

\section{Bargaining games}

We consider a dynamic game of perfect information $\Gamma$, where a set $N$ of players with cardinality $n \geq 3$ has to agree on the choice of a payoff vector in a set of feasible payoffs $V$. We use the notation $e_{0}$ for the vector $(0, \ldots, 0)$, and $e_{i}$ for the $i$-th unit vector in $\mathbb{R}^{N}$. We make four assumptions on the set of feasible payoffs $V$ :

1. $V$ is a closed subset of $\mathbb{R}_{+}^{N}$.

2. $\forall v \in V, \forall i \in N, v_{i} \leq 1$.

3. $e_{0} \in V, \forall i \in N, e_{i} \in V$.

4. $\forall v \in V$, the set $\left\{i \in N \mid v_{i}=1\right\}$ contains at most one point.

These four assumptions are satisfied in most applications and are not very restrictive. Closedness is a standard technical assumption. The second assumption is just a normalization of the set $V$. Any bounded set of feasible payoffs satisfies this assumption after an appropriate linear transformation of the payoffs. The first and second assumption together plus the standard assumption that the bargaining problem is essential implies that we can assume without loss of generality that for every $i \in N$ there is some $v \in V$ such that $v_{i}=1$. Together with the standard requirement that $V$ is comprehensive from below, this would imply the third assumption. The last assumption says that it is not possible for two players to reach their maximum utility level simultaneously. This assumption would be satisfied if players can make some transfers to each other. Notice that our assumptions permit the set $V$ to be non-convex or to be discrete.

The set of permutations from $\{1, \ldots, n\}$ into $N$ is denoted by $\Pi$. In each time period $t=0,1,2, \ldots$, nature selects the proposer and the order of responders by means of a permutation $\pi^{t} \in \Pi$. The numbers $\{1, \ldots, n\}$ are the positions of the players in the bargaining protocol. Player $\pi^{t}(1)$ makes a proposal $x^{t} \in V$, after which Player $\pi^{t}(2)$ responds to the proposal by accepting or rejecting it. If Player $\pi^{t}(2)$ accepts, it is Player $\pi^{t}(3)$ 's turn to respond to the proposal and so on. If all players accept the proposal, the game ends and the proposal is implemented. If one of the players rejects the proposal, the next period begins. In the next time period nature again selects a permutation, which determines the proposer and the order of the players to respond to the proposal. The utility of Player $i \in N$ who receives outcome $x_{i}$ in period $t$ is $\delta_{i}^{t} x_{i}$, where $\delta_{i} \in[0,1)$ is the discount factor for Player $i$. The utility of perpetual disagreement is 0 for all players.

Our bargaining protocol is very general. There are no restrictions on the process by which the permutation is selected in each round. The permutation is therefore selected in each round by means of a probability distribution over the set of all permutations which may depend on the entire history of play. We explain next how the protocols as described in Selten (1981), Herrero (1985), Binmore (1987), Chatterjee et al. (1993), Merlo and Wilson (1995), Banks and Duggan (2000), Kalandrakis (2006), and Herings and Predtetchinski (2010) follow as special cases.

In Selten (1981) and Chatterjee et al. (1993) there is an endogenous protocol for the players: the player who rejects is the next proposer. In this case the permutation in each period depends on the history of play in the previous period. Herrero (1985) considers a model with players that rotate in making offers. In our model, this is achieved by specifying $\pi^{t}(k)=$ $t+k \bmod n$. In Binmore (1987) and Banks and Duggan (2000) the proposer selection process is modeled by time-invariant recognition probabilities. The probability that Player $i \in N$ is recognized to make a proposal is constant across all periods. In Merlo and Wilson (1995), Kalandrakis (2006), and Herings and Predtetchinski (2010), the order in which players act in any period is determined by a Markov process: if the state of the game is $s$, then the game moves to state $s^{\prime}$ with probability $p\left(s, s^{\prime}\right)$. In other words, the current state depends on the previous state and actions of the players do not influence the state, a specification that is allowed for in our model. Our bargaining protocol even allows for the next proposer to depend on the current proposal, or on proposals made in the past, something which is not covered in the bargaining literature so far.

A history $h$ is a sequence of actions that have occurred before a particular decision node in the game. There are three different types of histories. Every period there are histories ending with a move by nature, there are histories ending with 
Table 1

The bargaining protocol in Example 3.1.

\begin{tabular}{|c|c|c|c|c|c|c|c|}
\hline & $t=0$ & $t=1$ & $t=2$ & $\ldots$ & $t=n-1$ & $t=n$ & $\ldots$ \\
\hline$\pi^{t}(1)$ & 1 & 2 & 3 & $\ldots$ & $n$ & 1 & $\ldots$ \\
\hline$\pi^{t}(2)$ & 2 & 3 & 4 & $\ldots$ & 1 & 2 & $\ldots$ \\
\hline$\pi^{t}(3)$ & 3 & 4 & 5 & $\ldots$ & 2 & 3 & $\ldots$ \\
\hline : & : & : & : & : & : & : & : \\
\hline$\pi^{t}(n)$ & $:$ & $:$ & $:$ & $:$ & $:$ & $:$ & $\cdot$ \\
\hline$\pi^{t}(n-1)$ & $n-1$ & $n$ & 1 & $\ldots$ & $n-2$ & $n-1$ & $\ldots$ \\
\hline$\pi^{t}(n)$ & $n$ & 1 & 2 & $\ldots$ & $n-1$ & $n$ & $\ldots$ \\
\hline
\end{tabular}

a move by the proposer, and there are histories ending with a move by a responder. Any non-terminal history is of one of the following three types:

1. $h \in H_{1}^{t}$ if and only if $h=\left(\pi^{0}, x^{0}, r^{0}, \ldots, \pi^{t-1}, x^{t-1}, r^{t-1}, \pi^{t}\right)$,

2. $h \in H_{2}^{t}$ if and only if $h=\left(\pi^{0}, x^{0}, r^{0}, \ldots, \pi^{t-1}, x^{t-1}, r^{t-1}, \pi^{t}, x^{t}\right)$,

3. $h \in H_{3}^{t}$ if and only if $h=\left(\pi^{0}, x^{0}, r^{0}, \ldots, \pi^{t-1}, x^{t-1}, r^{t-1}, \pi^{t}, x^{t}, r^{t}\right)$,

where, for $k=0, \ldots, t, \pi^{k}$ is the permutation of the set $N$ selected by nature in period $k, x^{k} \in V$ is the proposal in period $k$, and $r^{k} \in N \backslash\left\{\pi^{k}(1)\right\}$ is the player who rejected $x^{k}$. After a history $h \in H_{1}^{t}$ the proposer moves, after a history $h \in H_{2}^{t}$ a responder moves, and after a history $h \in H_{3}^{t}$ nature moves. In principle, one has to distinguish between histories after which the first responder moves, histories after which the second responder moves, and so on. We shall not make such a distinction. The symbol $h \in H_{2}^{t}$ might denote any of these histories. When Player $\pi^{t}(j)$ casts a vote after history $h$, it is to be understood that the Players $\pi^{t}(2), \ldots, \pi^{t}(j-1)$ have already accepted the proposal. Histories in $H_{1}^{t}$ are called proposer histories and those in $H_{2}^{t}$ responder histories. We denote the union of $H_{1}^{t}, H_{2}^{t}$, and $H_{3}^{t}$ over $t=0,1, \ldots$ by $H_{1}, H_{2}$, and $H_{3}$, respectively.

A strategy $\sigma_{i}$ of Player $i$ is a function that assigns a point in $V$ to each proposer history $h \in H_{1}$ ending with a permutation $\pi^{t}$ such that $\pi^{t}(1)=i$, and an element in \{accept, reject\} to each responder history $h \in H_{2}$ with the last permutation $\pi^{t}$ being such that $\pi^{t}(1) \neq i$. All our results make use of pure strategy profiles only.

A strategy profile is denoted by $\sigma=\left(\sigma_{i}\right)_{i \in N}$. Given a strategy profile $\sigma$ and a history $h \in H_{1}^{t}$, we use $p^{t}(h)$ for the resulting proposal.

A strategy profile $\sigma$ is said to have the immediate acceptance property if for every $t \geq 0$ and every history $h \in H_{1}^{t}$, the proposal $p^{t}(h)$ is accepted by all responders. If a strategy profile $\sigma$ has the immediate acceptance property, then the first proposal to be made is accepted, and the game ends in period 0.

A strategy $\sigma_{i}$ has K-recall if it assigns the same action to Player $i$ for all histories of the same length that coincide in the last $K$ periods. More precisely, a strategy $\sigma_{i}$ has $K$-recall if for all histories $h$ and $\bar{h}$ after which Player $i$ moves, it holds that $\sigma_{i}(h)=\sigma_{i}(\bar{h})$ whenever one of the following cases is true for some $t=0,1, \ldots$ :

1. $h, \bar{h} \in H_{1}^{t}$, for $k=t-K, \ldots, t-1: \pi^{k}=\bar{\pi}^{k}, x^{k}=\bar{x}^{k}, r^{k}=\bar{r}^{k}$, and $\pi^{t}=\bar{\pi}^{t}$;

2. $h, \bar{h} \in H_{2}^{t}$, for $k=t-K, \ldots, t-1: \pi^{k}=\bar{\pi}^{k}, x^{k}=\bar{x}^{k}, r^{k}=\bar{r}^{k}$, and $\pi^{t}=\bar{\pi}^{t}, x^{t}=\bar{x}^{t}$.

A strategy $\sigma_{i}$ has infinite recall if it does not have $K$-recall for any $K$.

A strategy profile $\sigma$ has $K$-recall if every strategy $\sigma_{i}, i \in N$, has $K$-recall. A strategy profile $\sigma$ has infinite recall if it does not have $K$-recall for any $K$.

In this paper we use subgame perfect equilibrium (SPE) as the solution concept. A strategy profile is a subgame perfect equilibrium if it induces a Nash equilibrium in every subgame of the original game.

\section{Herrero-Haller's example}

In this section we re-examine the example of an $n$-player divide-the-dollar game as studied in Herrero (1985). Herrero claims that given any division of the dollar, there is a subgame perfect equilibrium such that the given division is proposed and unanimously accepted in period zero. We quote Herrero's definition of the strategy profile and argue that it is incomplete. We next reproduce the proof of the claim using Hans Haller's definition of the strategy profile (private communication). We show that the strategies as defined by Haller have infinite recall. At the end of the section we briefly discuss Shaked's example of multiplicity of equilibria as reported in Binmore et al. (1992).

Example 3.1. Consider a game with $N=\{1, \ldots, n\}$ and $V=\left\{x \in \mathbb{R}_{+}^{n} \mid x_{1}+\cdots+x_{n} \leq 1\right\}$. The players have a common discount factor $\delta$. The game proceeds as in Section 2, with the sequence of moves given by the rule displayed in Table 1 .

Theorem 3.2. Consider the bargaining game of Example 3.1. If $1 /(n-1)<\delta<1$, then for every proposal a $\in V$, there exists an SPE in which at $t=0$ the proposal $a$ is made by Player 1 and accepted by Players $2, \ldots, n$. 
Proof. See Appendix A.

Herrero (1985) defines the following strategy profile in an attempt to prove Theorem 3.2.

1. Player 1 proposes $a$, which is unanimously accepted at time 0 .

2. $a$ is proposed and accepted at time $t$, provided $a$ was proposed at time $t-1$ (all $t>0$ ).

3. If Player $j$ deviates from (1) or (2) and proposes $y$ such that $y_{k}<\delta$ for some $k \neq j$ at time $\tau$, then

(a) Player $k$ rejects $y$

(b) $e_{k}$ is proposed and accepted at time $\tau+1$

(c) $e_{k}$ is proposed and accepted at time $t$, provided $e_{k}$ was proposed at time $t-1$ (all $t>\tau+1$ )

(d) and, if Player $l$ deviates from (b) or (c) to propose $z$ with $z_{h}<\delta$ for some $h \neq l$ at time $T$, then begin (a) again with $j=l$ and $k=h$.

Unfortunately, the above definition appears to be incomplete: if under item 3 there is more than one Player $k \neq j$ with $y_{k}<\delta$ it is not clear which one should be chosen. To illustrate this difficulty, consider a history where in period 0 Player 1 proposes $y=(\delta,(1-\delta) / 2,(1-\delta) / 2) \neq a$ and Player 2 accepts. It is now Player 3's turn to respond to the proposal. Notice that $y_{2}<\delta$ and $y_{3}<\delta$. Should Player 3 accept or reject the proposal $y$ ? This question cannot be answered unless it is decided whether the next proposal is going to be $e_{2}$ or $e_{3}$ if Player 3 rejects $y$.

It is clear that one can interpret Conditions $1-3$ in a number of ways. One such interpretation is due to Haller (private communication) and is reproduced below. For each $t \geq 0$, for each $x \in V$, we define

$$
i^{t+1}(x)=\min \left\{k \neq \pi^{t}(1) \mid x_{k} \leq \delta\right\} .
$$

This expression is well-defined since we assume that $\delta>1 /(n-1)$. We inductively define the sequence $s^{0}, s^{1}, \ldots$ of functions, where $s^{t}: V^{t} \rightarrow V$ with $V^{t}$ the $t$-fold product of $V$. We define $s^{0}=a$ and

$$
s^{t+1}\left(x^{0}, \ldots, x^{t}\right)= \begin{cases}s^{t}\left(x^{0}, \ldots, x^{t-1}\right), & \text { if } x^{t}=s^{t}\left(x^{0}, \ldots, x^{t-1}\right), \\ e_{i t+1}\left(x^{t}\right), & \text { if } x^{t} \neq s^{t}\left(x^{0}, \ldots, x^{t-1}\right) .\end{cases}
$$

The function $s^{t}$ represents the "state of mind" of the players in period $t$ and corresponds to the proposal that should be made in period $t$.

We define the strategy profile $\sigma$ as follows:

1. For each $t \geq 0$, for each history $h=\left(\pi^{0}, x^{0}, r^{0}, \ldots, \pi^{t-1}, x^{t-1}, r^{t-1}, \pi^{t}\right)$, Player $\pi^{t}(1)$ proposes $p^{t}(h)=s^{t}\left(x^{0}, \ldots, x^{t-1}\right)$.

2. After history $\left(\pi^{0}, x^{0}, r^{0}, \ldots, \pi^{t}, x^{t}\right)$, Player $\pi^{t}(k)$, where $k \in\{2, \ldots, n\}$, accepts $x^{t}$ if $\left[x^{t}=s^{t}\left(x^{0}, \ldots, x^{t-1}\right)\right]$ or $\left[i^{t+1}\left(x^{t}\right)=\right.$ $\pi^{t}(\ell)$ and $k>\ell$ ] and rejects otherwise.

The state of mind changes if the proposal is not equal to the current state of mind, irrespective of the fact whether the proposer demands a payoff greater or smaller than the corresponding coordinate of the current state of mind. After a change, the state of mind becomes the unit vector that gives a payoff of one to the responding player with the lowest index for whom a share less than or equal to $\delta$ was proposed. The responding players compare the proposal $x^{t}$ in period $t$ to the state of mind $s^{t}\left(x^{0}, \ldots, x^{t-1}\right)$ which should have been proposed in period $t$. In case the actual proposal is different, all players reject it, except when they vote after Player $i^{t+1}\left(x^{t}\right)$, in which case they accept. This will ensure that the criterion of subgame perfection is met. If the proposal $x^{t}$ in period $t$ is equal to $s^{t}\left(x^{0}, \ldots, x^{t-1}\right)$, then all responders accept.

In Appendix A, we give a proof of Theorem 3.2 by showing that the strategy profile $\sigma$ is a subgame perfect equilibrium provided that $\delta \in\left(\frac{1}{n-1}, 1\right)$.

We conclude our discussion of Herrero-Haller's construction by showing that the strategy profile $\sigma$ has infinite recall. Notice that the functions $s^{t}$ are being determined recursively, that is $s^{t+1}$ is obtained as a function of $s^{t}$ and the current proposal $x^{t}$. We now provide sufficient conditions for recursively defined functions to have infinite recall.

Let $X$ and $Y$ be sets with $Y \subset X$. For each $t \geq 1$, let $s^{t}: X^{t} \rightarrow Y$ be a function. We write $s=\left(s^{0}, s^{1}, \ldots\right)$. The sequence $s$ is said to have 0 -recall if each $s^{t}$ is a constant function. It is said to have $k$-recall for $k \geq 1$ if $s^{t}\left(x^{0}, \ldots, x^{t-1}\right)=s^{t}\left(\bar{x}^{0}, \ldots, \bar{x}^{t-1}\right)$ whenever $t \geq k$ and $x^{\ell}=\bar{x}^{\ell}$ for each $\ell \in\{t-k, \ldots, t-1\}$. The sequence is said to have infinite recall if it does not have $k$-recall for any $k \geq 0$.

Let $a$ be a point of $Y$, and, for each $t \geq 1$, let $f^{t}: X \times Y \rightarrow Y$ be a function. We define the sequence $s=\left(s^{0}, s^{1}, \ldots\right)$ of functions $s^{t}: X^{t} \rightarrow Y$ by induction on $t$ as follows:

$$
\begin{aligned}
& s^{0}=a, \\
& s^{t+1}\left(x^{0}, \ldots, x^{t}\right)=f^{t+1}\left(x^{t}, s^{t}\left(x^{0}, \ldots, x^{t-1}\right)\right), \quad t \geq 0 .
\end{aligned}
$$

Claim 3.3. Let $f=\left(f^{1}, f^{2}, \ldots\right)$ be a sequence of functions with, for $t \geq 1, f^{t}: X \times Y \rightarrow Y$ and $a \in Y$ be such that [1] $f^{t}(a, a)=a$ for each $t \geq 1$, [2] there is $t \geq 1$ and $x \in X$ such that $f^{t}(x, a) \neq a$, and [3] for each $t \geq 1$, for every $y, y^{\prime} \in Y$ such that $y \neq y^{\prime}$, there is $x \in X$ such that $f^{t}(x, y) \neq f^{t}\left(x, y^{\prime}\right)$. Then s as defined by (3.2)-(3.3) has infinite recall. 
Proof. The proof is by induction on $k$. We show that $s$ does not have 0 -recall. Suppose on the contrary that $s^{t}$ is a constant for each $t$. Then by [1] each $s^{t}$ is identically equal to $a$ on $X^{t}$. But for $t$ and $x$ as in Condition [2], we have that

$$
s^{t}(a, \ldots, a, x)=f^{t}\left(x, s^{t-1}(a, \ldots, a)\right)=f^{t}(x, a) \neq a,
$$

leading to a contradiction.

Assume $s$ does not have $k$-recall for some $k \geq 0$. Then there is $t \geq k$ and there are points $\left(x^{0}, \ldots, x^{t-1}\right),\left(\bar{x}^{0}, \ldots, \bar{x}^{t-1}\right) \in X^{t}$ such that $x^{\ell}=\bar{x}^{\ell}$ for each $\ell \in\{t-k, \ldots, t-1\}$, and

$$
s^{t}\left(x^{0}, \ldots, x^{t-1}\right) \neq s^{t}\left(\bar{x}^{0}, \ldots, \bar{x}^{t-1}\right) .
$$

It then follows by [3] that there is an $x^{t} \in X$ such that $f^{t+1}\left(x^{t}, s^{t}\left(x^{0}, \ldots, x^{t-1}\right)\right) \neq f^{t+1}\left(x^{t}, s^{t}\left(\bar{x}^{0}, \ldots, \bar{x}^{t-1}\right)\right)$, so

$$
s^{t+1}\left(x^{0}, \ldots, x^{t-1}, x^{t}\right) \neq s^{t+1}\left(\bar{x}^{0}, \ldots, \bar{x}^{t-1}, x^{t}\right),
$$

proving that $s$ does not have $k+1$-recall.

We now use the sufficient conditions of Claim 3.3 to show that the Herrero-Haller strategies have infinite recall.

Claim 3.4. The strategy profile $\sigma$ has infinite recall.

Proof. We prove the claim by showing that the sequence $s=\left(s^{0}, s^{1}, \ldots\right)$ of states of mind as defined by Equation (3.1) has infinite recall. We take $X=V$ and $Y=\left\{e_{1}, \ldots, e_{n}, a\right\}$ and, for $t=1, \ldots, n$, we define the function $f^{t}: X \times Y \rightarrow Y$ by

$$
f^{t}(x, y)= \begin{cases}y, & \text { if } x=y, \\ e_{i^{t}(x)}, & \text { otherwise, }\end{cases}
$$

where $i^{t}(x)=\min \left\{k \neq t \mid x_{k} \leq \delta\right\}$. For $k \in \mathbb{N}$ and $t=1, \ldots, n$, we define $f^{t+n k}=f^{t}$. We verify next that the functions $f^{t}$ thus defined satisfy the conditions of Claim 3.3.

Condition [1] is immediate. To verify Condition [2], we argue as follows. If $a$ is not a member of $\left\{e_{1}, \ldots, e_{n}\right\}$, then $f^{1}\left(e_{1}, a\right)=e_{2} \neq a$. If $a=e_{t}$ for some $t \in\{1, \ldots, n\}$, take any $i \in\{1, \ldots, n\} \backslash\{t\}$ and notice that $f^{t}\left(e_{i}, e_{t}\right)=e_{i^{t}}\left(e_{i}\right) \neq e_{t}$ because $i^{t}\left(e_{i}\right) \neq t$.

To verify condition [3], consider $t \geq 1$ and $y, y^{\prime} \in Y$ with $y \neq y^{\prime}$. We have $f^{t}(y, y)=y$ and $f^{t}\left(y, y^{\prime}\right)=e_{i^{t}(y)}$. Suppose $y=e_{i^{t}(y)}$ and define $j=i^{t}(y)$. Since $i^{t}\left(e_{j}\right) \neq j$, we have that

$$
e_{j}=y=e_{i t\left(e_{j}\right)} \neq e_{j},
$$

a contradiction.

A well-known example of multiplicity of subgame perfect equilibria in a multilateral bargaining game is reported in Binmore et al. (1992, pp. 191-192), where the example is attributed to Shaked. It is argued that in a three-player dividethe-dollar game with rotating proposers, any division of the dollar can be supported as a subgame perfect equilibrium outcome. The strategies are built using states of mind similar to the functions $s^{t}$ in Herrero-Haller's construction. One can show, in much the same way as has been done above for Herrero-Haller's construction, that the strategies given in Binmore, Osborne, and Rubinstein have infinite recall. We refer to Herings et al. (2012), the working paper version of this paper, for the details.

\section{Illustration of the main result}

Consider a three-player bargaining game where only Player 1 can make proposals. Intuition seems to tell us that such a game is hardly of any interest: since Player 1 has all the bargaining power, surely the only outcome that can arise in this game is the one where Player 1 gets the entire surplus. We show this intuition to be wrong. In fact, there exists a subgame perfect equilibrium where Player 2 gets the entire surplus and one where Player 3 does. Furthermore, there is even a subgame perfect equilibrium where the zero vector $(0,0,0)$ is proposed and accepted. Unlike the examples of the preceding sections, the subgame perfect equilibrium constructed below has one-period recall. To compute his proposal in a given period, Player 1 only has to remember his previous proposal and whether it was Player 2 or 3 who rejected it. The responses of Players 2 and 3 only depend on the current proposal.

While these claims are subsumed by our main theorem in the next section, we offer an explicit proof. We do so to illustrate the construction in the next section.

Example 4.1. Let $N=\{1,2,3\}$ and $V=\left\{x \in \mathbb{R}_{+}^{3} \mid x_{1}+x_{2}+x_{3} \leq 1\right\}$. The bargaining game proceeds as described in Section 2 , where the period $t$ permutation $\pi^{t}$ is given by $\pi^{t}(i)=i$ for every $t$. So every period, Player 1 makes the proposal, after which Player 2 responds. If Player 2 accepts, it is Player 3's turn to respond to the proposal. The players have a common discount factor $\delta$. We define $U=\left\{e_{0}, e_{2}, e_{3}\right\}$. 
Theorem 4.2. Consider the bargaining game of Example 4.1. If $1 / 2<\delta<1$, then for every a $\in U$ there exists a one-period recall SPE such that in period 0 a is proposed by Player 1 and accepted by Players 2 and 3.

Since the permutation $\pi^{t}$ is fixed throughout the game, we omit it from the notation for histories. The strategy profile $\sigma$ is defined as follows.

1. At $t=0$, Player 1 proposes $a$. For each $t \geq 1$, after history $h=\left(x^{0}, r^{0}, \ldots, x^{t-1}, r^{t-1}\right)$, Player 1 proposes

$$
p^{t}(h)= \begin{cases}e_{0}, & \text { if } \quad\left[x^{t-1} \in U\right], \\ e_{2}, & \text { if } \quad\left[x^{t-1} \notin U \text { and } r^{t-1}=2\right], \\ e_{3}, & \text { if } \quad\left[x^{t-1} \notin U \text { and } r^{t-1}=3\right] .\end{cases}
$$

2. For $t \geq 0$, after history $\left(x^{0}, r^{0}, \ldots, x^{t-1}, r^{t-1}, x^{t}\right)$, Player 2 accepts $x^{t}$ if $x^{t} \in U$, and rejects otherwise.

3. For $t \geq 0$, after history $\left(x^{0}, r^{0}, \ldots, x^{t-1}, r^{t-1}, x^{t}\right)$, Player 3 accepts $x^{t}$ if $x^{t} \in U$ or $x_{3}^{t} \geq \delta$, and rejects otherwise.

According to the strategy profile, the proposal $a \in U$ is made and accepted in period 0 . Any proposal outside $U$ is rejected by Player 2, after which proposal $e_{2}$ is made and accepted in the next period.

The intuition behind the strategies is the following. Player 1 is indifferent between any of his actions, because any proposal that Player 2 accepts gives him a payoff equal to 0 . Player 2 is encouraged to accept proposals from $U$ and to reject other proposals: after the rejection of a proposal that is not from $U, e_{2}$ will be proposed and accepted. The same logic works for Player 3 except that he also accepts proposals that give him a payoff higher than or equal to $\delta$. Notice that unlike the construction used in Section 3, where every responder accepts when offered a payoff greater than or equal to $\delta$, it is enough to have the last responder accept when offered such a payoff.

Rubinstein (1982) has shown that there is a unique subgame perfect equilibrium for the two-player case. Our approach indeed breaks down for the case with two players. Subgame perfection requires the last player who responds to a proposal offering a payoff of at least $\delta$ to accept. In the two-player case, this enables the proposer to obtain at least a payoff of $1-\delta$, and a contraction argument can be used to pin down equilibrium payoffs to a unique value. In cases with three or more players, a proposer cannot make a proposal that guarantees him a strictly positive payoff, as the proposer cannot offer more than $\delta$ to all the responders if $\delta$ is sufficiently high.

Claim 4.3. The strategy profile $\sigma$ has the immediate acceptance property. If $1 / 2<\delta<1$, the strategy profile $\sigma$ is an SPE.

Proof. The profile $\sigma$ has the immediate acceptance property because Player 1 only makes proposals that belong to $U$ and all members of $U$ are accepted by Players 2 and 3. We verify that no player has a profitable one-shot deviation.

STEP 1. We show that Player 1 has no profitable one-shot deviations. At each history $h \in H_{1}^{t}$ any of Player 1 's actions yields Player 1 a payoff of zero. This follows because the only proposals accepted by Player 2 are $e_{0}, e_{2}$, and $e_{3}$, and each of these proposals gives payoff 0 to Player 1 . Hence Player 1 is indifferent between any of his actions. In particular, Player 1 has no profitable deviations from $\sigma$.

STEP 2. We show that Players 2 and 3 have no profitable one-shot deviations. Take a history $h \in H_{2}^{t}$ ending with the proposal $x^{t}$. We consider three cases:

1. $x^{t} \in U$,

2. $x^{t} \notin U$ and $x_{3}^{t}<\delta$,

3. $x^{t} \notin U$ and $x_{3}^{t} \geq \delta$.

Case 1: $x^{t} \in U$.

The strategy profile $\sigma$ calls for the acceptance of $x^{t}$. If Player 2 or Player 3 deviates and rejects, the proposal $e_{0}$ is made and accepted next period. Clearly, rejection is not a profitable deviation for the responding players.

Case 2: $x^{t} \notin U$ and $x_{3}^{t}<\delta$.

Consider Player 3. The strategy profile $\sigma$ recommends that Player 3 rejects. Rejection gives Player 3 a payoff of $\delta^{t+1}$, because the proposal $e_{3}$ is made and accepted in period $t+1$. If Player 3 deviates and accepts the proposal, he obtains payoff $\delta^{t} x_{3}^{t}<\delta^{t+1}$. So acceptance is not a profitable deviation.

Consider Player 2. The strategy profile $\sigma$ calls for Player 2 to reject. Rejection gives Player 2 a payoff of $\delta^{t+1}$, because the proposal $e_{2}$ is made and accepted next period. Suppose now Player 2 deviates and accepts $x^{t}$. Then Player 3 rejects $x^{t}$ and in period $t+1$ the proposal $e_{3}$ is made and accepted, leaving Player 2 with payoff zero. So acceptance is not a profitable deviation for Player 2.

Case 3: $x^{t} \notin U$ and $x_{3}^{t} \geq \delta$

Consider Player 3. According to $\sigma$, Player 3 should accept. Acceptance gives Player 3 the payoff $\delta^{t} x_{3}^{t} \geq \delta^{t+1}$. If Player 3 deviates and rejects the proposal, the proposal $e_{3}$ is made and accepted in period $t+1$, yielding Player 3 the payoff $\delta^{t+1}$. So rejection is not a profitable deviation. 
Consider Player 2. According to the strategy profile $\sigma$, Player 2 must reject. Rejection gives Player 2 a payoff of $\delta^{t+1}$, because the proposal $e_{2}$ is made and accepted in period $t+1$. Suppose now Player 2 deviates and accepts. Since Player 3 accepts $x^{t}$, Player 2 then receives a payoff of $\delta^{t} x_{2}^{t}$. Now $x_{2}^{t} \leq 1-x_{3}^{t} \leq 1-\delta \leq \delta$, so $\delta^{t} x_{2}^{t} \leq \delta^{t+1}$. So acceptance is not a profitable deviation for Player 2.

Our main result generalizes the example above in a number of ways. As has been discussed in Section 2 , we allow nature's choice of the permutations to be probabilistic and history dependent, where the choice of nature may even depend on aspects of the history of more than one period ago. Secondly, we only impose rather minimal assumptions on the set $V$ of feasible payoffs and we allow for heterogeneous discount factors. And finally, we show that any vector in the set $V$, not just the unit vectors, can be supported as a subgame perfect equilibrium outcome.

\section{The folk theorem for bargaining games}

In this section we prove our main result: if the players are sufficiently patient, given any point $a$ of the set $V$, one can find a subgame perfect equilibrium having one-period recall such that on the equilibrium path of play the point $a$ is proposed and accepted in period zero. As a corollary to this result, we show that there exists a subgame perfect equilibrium with one-period recall leading to perpetual disagreement. As a first step, we identify the level of patience needed for the result to hold.

Lemma 5.1. There exists $\bar{\delta}<1$ such that for every $v \in V$ the set $\left\{i \in N: v_{i} \geq \bar{\delta}\right\}$ contains at most one point.

Proof. Suppose not. Then there exists $i, j \in N$ with $i \neq j$ and a sequence $\left(v^{m}\right)_{m \in \mathbb{N}}$ of points in $V$ such that $v_{i}^{m} \geq 1-1 / m$ and $v_{j}^{m} \geq 1-1 / m$. Since the set $V$ is compact, we can assume without loss of generality that $v^{m}$ converges to $\bar{v} \in V$. But then $\bar{v}_{i} \geq 1$ and $\bar{v}_{j} \geq 1$, contradicting Assumption 4 .

For the rest of this section, we fix some $\bar{\delta}<1$ such that Lemma 5.1 is satisfied. We are now in a position to state our main result.

Theorem 5.2. Given any point $a \in V$ there exists a strategy profile $\sigma$ such that:

[1] The proposal $a$ is made in period 0 and is unanimously accepted. Furthermore, $\sigma$ has the immediate acceptance property.

[2] The strategy profile $\sigma$ has 1-period recall.

[3] If for every $i \in N$ it holds that $\delta_{i} \geq \bar{\delta}$, then $\sigma$ is an SPE.

We define our strategy profile by specifying actions for a player who is in a particular position in the game. There are $n$ positions in the game: the proposer, the player who responds first, the player who responds second, and so on. The strategy for the proposer, $\pi^{t}(1)$, specifies the proposal at each period $t$, as a function $p^{t}(h)$ of the history of play up to $t$. The strategies for "the player who responds first", Player $\pi^{t}(2)$, "the player who responds second", Player $\pi^{t}(3), \ldots$, and "the player who responds last", Player $\pi^{t}(n)$, specify their reaction to the proposal contingent on the history of play up to $t$.

We define $E=\left\{e_{0}\right\} \cup\left\{e_{i} \mid i \in N\right\}$. We define the strategy profile $\sigma$ as follows:

1. In period $t=0$, Player $\pi^{0}(1)$ proposes $p^{0}=a$. At $t=1$, after history $h=\left(\pi^{0}, x^{0}, r^{0}, \pi^{1}\right)$, Player $\pi^{1}(1)$ proposes

$$
p^{1}(h)=\left\{\begin{array}{lll}
e_{0}, & \text { if } \quad x^{0}=a \\
e_{r^{0}}, & \text { if } \quad x^{0} \neq a
\end{array}\right.
$$

For each $t \geq 2$, after history $h=\left(\pi^{0}, x^{0}, r^{0}, \ldots, \pi^{t-1}, x^{t-1}, r^{t-1}, \pi^{t}\right)$, Player $\pi^{t}(1)$ proposes

$$
p^{t}(h)= \begin{cases}e_{0}, & \text { if } x^{t-1} \in E, \\ e_{r^{t-1}}, & \text { if } x^{t-1} \notin E .\end{cases}
$$

2. After history $\left(\pi^{0}, x^{0}, r^{0}, \ldots, \pi^{t-1}, x^{t-1}, r^{t-1}, \pi^{t}, x^{t}\right)$, Player $\pi^{t}(j), j \in\{2, \ldots, n-1\}$, accepts $x^{t}$ if

- $\left[x^{t}=p^{t}\left(\pi^{0}, x^{0}, r^{0}, \ldots, \pi^{t}\right)\right]$ or

- $\left[t \geq 1\right.$ and $x^{t} \in E$ and $\left.x^{t} \neq e_{\pi^{t}(1)}\right]$,

and rejects otherwise.

3. After history $\left(\pi^{0}, x^{0}, r^{0}, \ldots, \pi^{t-1}, x^{t-1}, r^{t-1}, \pi^{t}, x^{t}\right)$, Player $\pi^{t}(n)$ accepts $x^{t}$ if

- $\left[x^{t}=p^{t}\left(\pi^{0}, x^{0}, r^{0}, \ldots, \pi^{t}\right)\right]$ or

- $\left[t \geq 1\right.$ and $x^{t} \in E$ and $\left.x^{t} \neq e_{\pi^{t}(1)}\right]$ or

- $\left[x_{\pi^{t}(n)}^{t} \geq \delta_{\pi^{t}(n)}\right]$,

and rejects otherwise. 
One interesting feature of our construction is that it is independent of the bargaining protocol, i.e. of the process by which nature chooses permutations of the players. Notice that the action to be taken at a particular history of the game, as specified above, does not depend on how nature's choices are generated.

Another feature of our construction is that after a given proposer history, the same proposal is to be made, irrespective of who the proposer is. Similarly, after any given responder history, the second responder is to respond to the proposal in the same way, irrespective of who the second responder is. Only the response of the last responder depends on his identity through the discount factor.

Our treatment of the last responder is slightly different from that of the other responders. The last responder is required to accept any proposal that offers him an amount at least equal to his discount factor, while this is not required for the other responders. This is caused by the fact that when the last responder casts his vote, he knows that all other responders have already accepted the proposal. Thus a yes vote by the last responder guarantees that the proposal will be implemented. This is not the case for the other responders.

Part [1] of Theorem 5.2 is immediate from the definition above: indeed, for every $h \in H_{1}^{t}$ the proposal $p^{t}(h)$ is accepted by all the responders. In particular, the period 0 proposal $p^{0}=a$ is unanimously accepted.

Part [2] is equally straightforward: Notice that every proposer history in period $t \geq 1$ is of the form $\left(h, x^{t-1}, r^{t-1}, \pi^{t}\right)$ where $h \in H_{1}^{t-1}$ is some proposer history in period $t-1$, while a responder history at $t$ can be written as $\left(h, x^{t-1}, r^{t-1}, \pi^{t}, x^{t}\right)$ where $h \in H_{1}^{t-1}$. It is clear from the definition above that the proposal $p^{t}\left(h, x^{t-1}, r^{t-1}, \pi^{t}\right)$ at the proposal history $\left(h, x^{t-1}, r^{t-1}, \pi^{t}\right)$ does not depend on $h$. And hence also the response $\sigma_{i}\left(h, x^{t-1}, r^{t-1}, \pi^{t}, x^{t}\right)$ of Player $i \neq \pi^{t}(1)$ at the responder history $\left(h, x^{t-1}, r^{t-1}, \pi^{t}, x^{t}\right.$ ) does not depend on $h$. It follows that the strategy profile $\sigma$ has one-period recall.

We proceed to prove part [3] of the theorem. The intuition behind our construction could be summarized as follows. According to $\sigma$, the responders only accept a proposal $p^{t}(h)$ as prescribed by the strategy profile $\sigma$, along with those unit vectors which give 0 to the proposer. This implies that any deviation by a proposer can only result in a payoff of zero, the essence of Claim 5.3 below. Rejections of proposals in the set $E$ are penalized by having the next proposer offer the zero vector $e_{0}$. Rejections of proposals outside the set $E$ are rewarded by having the next proposer offer the maximum payoff of 1 to the rejector.

The proof of part [3] of Theorem 5.2 is divided into three steps. Claim 5.3 shows that for each proposer history $h \in H_{1}^{t}$ any proposal other than $p^{t}(h)$ yields the proposer a payoff of zero. This implies in particular that no proposer has a profitable one-shot deviation from $\sigma$. Claim 5.4 shows that there are no profitable one-shot deviations from $\sigma$ at the responder histories in period 0 , and Claim 5.5 that there are no such deviations at period $t \geq 1$. In Claims 5.4 and 5.5 we assume that for every $i \in N, \delta_{i} \geq \bar{\delta}$.

Claim 5.3. Consider a proposer history $h \in H_{1}^{t}$. If a proposal $x^{t} \neq p^{t}(h)$ is made after history $h$, it leads to a payoff of 0 for the proposer. In particular, there are no profitable one-shot deviations from $\sigma$ at $h$.

Proof. Suppose the history $h$ ends with the permutation $\pi^{t}$. Take any $x^{t} \neq p^{t}(h)$. We consider two cases depending on whether $t=0$ or $t \geq 1$.

- Case $t=0$. We have $p^{0}=a$. The proposal $x^{0} \neq a$ is rejected by Player $\pi^{0}(2)$, and in period 1 the proposal $e_{\pi^{0}(2)}$ will be made and accepted, see part [1] of Theorem 5.2. This leads to payoff 0 for Player $\pi^{0}(1)$.

- Case $t \geq 1$. We consider three subcases:

1. Case $x^{t}=e_{\pi^{t}(1)}$. Since $x^{t} \neq p^{t}(h)$, the proposal $x^{t}$ is rejected by Player $\pi^{t}(2)$. Since the period $t+1$ proposal $e_{0}$ is accepted by part [1] of Theorem 5.2, Player $\pi^{t}(1)$ receives payoff 0 .

2. Case $x^{t} \in E \backslash\left\{e_{\pi^{t}(1)}\right\}$. In this case the proposal $x^{t}$ is unanimously accepted. Player $\pi^{t}(1)$ receives payoff 0 .

3. Case $x^{t} \notin E$. In this case the proposal $x^{t}$ is rejected by Player $\pi^{t}(2)$. The next proposal is $e_{\pi^{t}(2)}$, which is accepted. This leads to payoff 0 for Player $\pi^{t}(1)$.

In each case Player $\pi^{t}(1)$ receives a payoff of 0 .

Claim 5.4. Consider a responder history $h \in H_{2}^{0}$. If, for every $i \in N, \delta_{i} \geq \bar{\delta}$, then there are no profitable one-shot deviations from $\sigma$ at $h$.

Proof. Let $h=\left(\pi^{0}, x^{0}\right)$. We consider the following three cases:

1. $x^{0}=a$,

2. $x^{0} \neq a$ and $x_{\pi^{0}(n)}^{0} \geq \delta_{\pi^{0}(n)}$,

3. $x^{0} \neq a$ and $x_{\pi^{0}(n)}^{0}<\delta_{\pi^{0}(n)}$.

Case 1: $x^{0}=a$. Consider Player $\pi^{0}(j)$ where $j \in\{2, \ldots, n\}$. The strategy profile $\sigma$ prescribes that the proposal $x^{0}$ be accepted. Suppose Player $\pi^{0}(j)$ rejects $x^{0}$ instead. Then in period 1 the proposal $e_{0}$ will be made and accepted. Since 0 is the lowest payoff in the game, rejection is not a profitable deviation. 
Case 2: $x^{0} \neq a$ and $x_{\pi^{0}(n)}^{0} \geq \delta_{\pi^{0}(n)}$.

Consider Player $\pi^{0}(n)$. According to $\sigma$, Player $\pi^{0}(n)$ accepts $x^{0}$ and receives a payoff of $x_{\pi^{0}(n)}^{0} \geq \delta_{\pi^{0}(n)}$. If Player $\pi^{0}(n)$ deviates and rejects, in period 1 the proposal $e_{\pi^{0}(n)}$ is made and accepted. It leads to a payoff of $\delta_{\pi^{0}(n)}$ for Player $\pi^{0}(n)$, so the deviation is not profitable.

Consider Player $\pi^{0}(n-1)$. According to $\sigma$, Player $\pi^{0}(n-1)$ rejects $x^{0}$, the proposal $e_{\pi^{0}(n-1)}$ is made and accepted next period, and Player $\pi^{0}(n-1)$ receives payoff $\delta_{\pi^{0}(n-1)}$. Suppose now Player $\pi^{0}(n-1)$ deviates and accepts $x^{0}$. Since, according to $\sigma$, Player $\pi^{0}(n)$ accepts $x^{0}$, Player $\pi^{0}(n-1)$ receives the payoff $x_{\pi^{0}(n-1)}^{0}$. Now since $x^{0}$ belongs to $V$ and $x_{\pi^{0}(n)}^{0} \geq \delta_{\pi^{0}(n)} \geq \bar{\delta}$, we have $x_{\pi^{0}(n-1)}^{0}<\bar{\delta} \leq \delta_{\pi^{0}(n-1)}$ by the choice of $\bar{\delta}$. So the deviation is not profitable.

Consider Player $\pi^{0}(j)$ where $j \in\{2, \ldots, n-2\}$. According to the strategy profile $\sigma$, Player $\pi^{0}(j)$ rejects $x^{0}$, and the proposal $e_{\pi^{0}(j)}$ is made and accepted next period. This leads to a payoff of $\delta_{\pi^{0}(j)}$. Suppose now Player $\pi^{0}(j)$ deviates and accepts $x^{0}$. Then $x^{0}$ will be rejected by Player $\pi^{0}(j+1)$, and the proposal $e_{\pi^{0}(j+1)}$ is made and accepted next period. This leads to a payoff of zero to Player $\pi^{0}(j)$. So the deviation is not profitable.

Case 3: $x^{0} \neq a$ and $x_{\pi^{0}(n)}^{0}<\delta_{\pi^{0}(n)}$.

Consider Player $\pi^{0}(n)$. According to $\sigma$, Player $\pi^{0}(n)$ rejects $x^{0}$, and the proposal $e_{\pi^{0}(n)}$ is made and accepted in period 1 , leading to a payoff of $\delta_{\pi^{0}(n)}$ to Player $\pi^{0}(n)$. If Player $\pi^{0}(n)$ deviates and accepts, he receives only $x_{\pi^{0}(n)}^{0}<\delta_{\pi^{0}(n)}$, so the deviation is not profitable.

Consider Player $\pi^{0}(j)$ where $j \in\{2, \ldots, n-1\}$. According to $\sigma$, Player $\pi^{0}(j)$ rejects $x^{0}$, and the proposal $e_{\pi^{0}(j)}$ is made and accepted in period 1, leading to a payoff of $\delta_{\pi^{0}(j)}$ for Player $\pi^{0}(j)$. Suppose Player $\pi^{0}(j)$ deviates and accepts $x^{0}$. Then $x^{0}$ will be rejected by Player $\pi^{0}(j+1)$ and next period the proposal $e_{\pi^{0}(j+1)}$ will be made and accepted, thus leaving Player $\pi^{0}(j)$ with a payoff of 0 . Thus the deviation is not profitable.

Claim 5.5. Consider a responder history $h \in H_{2}^{t}$ where $t \geq 1$. If, for every $i \in N, \delta_{i} \geq \bar{\delta}$, then there are no profitable one-shot deviations from $\sigma$ at $h$.

Proof. The history $h$ is of the form $h=\left(h^{\prime}, \pi^{t}, x^{t}\right)$ where $h^{\prime} \in H_{3}^{t-1}, \pi^{t}$ is the permutation chosen by nature at $h^{\prime}$, and $x^{t}$ is the proposal chosen by Player $\pi^{t}(1)$ at $\left(h^{\prime}, \pi^{t}\right)$. We consider the following cases:

1. $x^{t} \in E \backslash\left\{e_{\pi^{t}(1)}\right\}$,

2. $x^{t}=e_{\pi^{t}(1)}$,

3. $x^{t} \notin E$ and $x_{\pi^{t}(n)}^{t} \geq \delta_{\pi^{t}(n)}$,

4. $x^{t} \notin E$ and $x_{\pi^{t}(n)}^{t}<\delta_{\pi^{t}(n)}$.

Case 1: $x^{t} \in E \backslash\left\{e_{\pi^{t}(1)}\right\}$.

According to the strategy profile $\sigma$, all the responders $\pi^{t}(j)$ for $j \in\{2, \ldots, n\}$ accept $x^{t}$. In case of a rejection, next period the proposal $e_{0}$ is made and accepted. Since 0 is the lowest possible payoff in the game, a deviation is not profitable.

Case 2: $x^{t}=e_{\pi^{t}(1)}$.

Either action of Player $\pi^{t}(j)$ for $j \in\{2, \ldots, n\}$ results in a payoff of zero. Indeed, if the proposal $x^{t}$ is accepted, Player $\pi^{t}(j)$ receives $x_{\pi^{t}(j)}^{t}=0$. And if $x^{t}$ is rejected, next period the proposal will be $e_{0}$, which is accepted. In either case Player $\pi^{t}(j)$ receives payoff 0 . It follows that there are no profitable one-shot deviations from $\sigma$ at $h$.

Case 3: $x^{t} \notin E$ and $x_{\pi^{t}(n)}^{t} \geq \delta_{\pi^{t}(n)}$.

Notice that $x^{t} \neq p^{t}\left(h^{\prime}, \pi^{t}\right)$ because for $t \geq 1$ the proposal $p^{t}\left(h^{\prime}, \pi^{t}\right)$ belongs to $E$.

Consider Player $\pi^{t}(n)$. According to $\sigma$, Player $\pi^{t}(n)$ accepts $x^{t}$ and receives a payoff of $\delta_{\pi^{t}(n)}^{t} x_{\pi^{t}(n)}^{t} \geq \delta_{\pi^{t}(n)}^{t+1}$. If Player $\pi^{t}(n)$ deviates and rejects, in period $t+1$ the proposal $e_{\pi^{t}(n)}$ is made and accepted. This leads to a payoff of $\delta_{\pi^{t}(n)}^{t+1}$ for Player $\pi^{t}(n)$, so the deviation is not profitable.

Consider Player $\pi^{t}(n-1)$. According to $\sigma$, Player $\pi^{t}(n-1)$ rejects $x^{t}$, the proposal $e_{\pi^{t}(n-1)}$ is made and accepted next period, and Player $\pi^{t}(n-1)$ receives payoff $\delta_{\pi^{t}(n-1)}^{t+1}$. Suppose now Player $\pi^{t}(n-1)$ deviates and accepts $x^{t}$. Since according to $\sigma$, Player $\pi^{t}(n)$ accepts $x^{t}$, Player $\pi^{t}(n-1)$ then receives the payoff $\delta_{\pi^{t}(n-1)}^{t} x_{\pi^{t}(n-1)}^{t}$. Now since $x^{t}$ is a point of $V$ and $x_{\pi^{t}(n)}^{t} \geq \delta_{\pi^{t}(n)} \geq \bar{\delta}$, we have $x_{\pi^{t}(n-1)}^{t}<\bar{\delta} \leq \delta_{\pi^{t}(n-1)}$, by the choice of $\bar{\delta}$. Hence $\delta_{\pi^{t}(n-1)}^{t} x_{\pi^{t}(n-1)}^{t}<\delta_{\pi^{t}(n-1)}^{t+1}$ and the deviation is not profitable.

Consider Player $\pi^{t}(j)$ where $j \in\{2, \ldots, n-2\}$. According to the strategy profile $\sigma$, Player $\pi^{t}(j)$ rejects $x^{t}$, and the proposal $e_{\pi^{t}(j)}$ is made and accepted next period. This leads to a payoff of $\delta_{\pi^{t}(j)}^{t+1}$. Suppose now Player $\pi^{t}(j)$ deviates and accepts $x^{t}$. Then $x^{t}$ will be rejected by Player $\pi^{t}(j+1)$, and next period the proposal $e_{\pi^{t}(j+1)}$ will be made and accepted. This leads to a payoff of zero to Player $\pi^{t}(j)$. So the deviation is not profitable.

Case 4: $x^{t} \notin E$ and $x_{\pi^{t}(n)}^{t}<\delta_{\pi^{t}(n)}$. 
Notice that $x^{t}$ is not equal to $p^{t}\left(h^{\prime}, \pi^{t}\right)$ since for $t \geq 1$ the latter is an element of $E$. Consider Player $\pi^{t}(n)$. According to $\sigma$, Player $\pi^{t}(n)$ rejects, and the proposal $e_{\pi^{t}(n)}$ is made and accepted in period $t+1$, leading to a payoff of $\delta_{\pi^{t}(n)}^{t+1}$ to Player $\pi^{t}(n)$. If Player $\pi^{t}(n)$ deviates and accepts, he receives only a payoff of $\delta_{\pi^{t}(n)}^{t} x_{\pi^{t}(n)}^{t}<\delta_{\pi^{t}(n)}^{t+1}$, so the deviation is not profitable. Consider Player $\pi^{t}(j)$ where $j \in\{2, \ldots, n-1\}$. According to $\sigma$, Player $\pi^{t}(j)$ rejects, and the proposal $e_{\pi^{t}(j)}$ is made and accepted in period $t+1$, leading to a payoff of $\delta_{\pi^{t}(j)}^{t+1}$ for Player $\pi^{t}(j)$. Suppose Player $\pi^{t}(j)$ deviates and accepts. Then $x^{t}$ will be rejected by Player $\pi^{t}(j+1)$ and the proposal $e_{\pi^{t}(j+1)}$ will be made and accepted in period $t+1$, thus leaving Player $\pi^{t}(j)$ with a payoff of 0 . Thus the deviation is not profitable.

We derive, as a corollary to our main result, that perpetual delay can also be sustained as a subgame perfect equilibrium outcome using a one-period recall strategy profile.

Corollary 5.6. There exists a strategy profile $\tau$ such that:

[1] On the path of play of $\tau$, the zero vector $e_{0}$ is proposed and is rejected in each round of bargaining, irrespective of the moves by nature. Thus $\tau$ leads to perpetual disagreement.

[2] The strategy profile $\tau$ has 1-period recall.

[3] If $\delta_{i} \geq \bar{\delta}$ for every $i \in N$, then $\tau$ is an SPE.

The strategy profile $\tau$ with the required properties is obtained by a modification of the strategy profile $\sigma$. Let $\sigma$ be the strategy profile as before with $a=e_{0}$. We let $\tau$ agree with $\sigma$ on all histories except possibly those responder histories where the responders react to the proposal $e_{0}$. Under $\tau$ the proposal $e_{0}$ is always to be rejected. More formally, define $\tau$ as follows:

1. For every proposer history $h=\left(\pi^{0}, \ldots, \pi^{t}\right)$, let $\tau_{\pi^{t}(1)}(h)=\sigma_{\pi^{t}(1)}(h)$. That is, the proposal under $\tau$ at $h$ equals the one under $\sigma$.

2. Consider a responder history $h=\left(\pi^{0}, x^{0}, \ldots, \pi^{t}, x^{t}\right)$. For each responder $i \in\left\{\pi^{t}(2), \ldots, \pi^{t}(n)\right\}$, let $\tau_{i}(h)=$ accept if and only if $\sigma_{i}(h)=$ accept and $x^{t} \neq e_{0}$.

Part [2] of the corollary follows since $\sigma$ has one-period recall.

Under the strategy profile $\tau$, the vector $e_{0}$ is proposed in period $t=0$. Furthermore, under the strategy profile $\tau$, the proposal $e_{0}$ is always rejected, and, as follows by the definition of $\sigma$, the next proposal is again $e_{0}$, irrespective of the chosen permutation. Thus, under the strategy profile $\tau$, the vector $e_{0}$ is proposed and rejected in every period, leading to perpetual disagreement irrespective of the moves by nature. This establishes part [1] of the corollary.

Recall that under the strategy profile $\sigma$, the period $t=0$ proposal $e_{0}$ is accepted. The next claim says that, even though the two strategy profiles might lead to different outcomes, they induce the same expected payoffs conditional on any history.

\section{Claim 5.7. Let $h \in H$ be any history. The vector of expected payoffs conditional on history $h$ under $\tau$ equals that under $\sigma$.}

Proof. First let $h$ be a responder history such that (a) the responders have to react to the proposal $e_{0}$, and (b) under the strategy profile $\sigma$ the proposal is accepted. Under the strategy profile $\sigma$, the vector of expected payoffs conditional on $h$ is obviously the zero vector. On the other hand, under the strategy profile $\tau$ the proposal $e_{0}$ is rejected, and is followed by the proposal $e_{0}$ which is again rejected, and so on. Thus the play under $\tau$ results in perpetual disagreement. Therefore, the vector of expected payoffs conditional on $h$ under $\tau$ is also zero. It is now easy to see that the result is true for any history $h$.

Now we argue that $\tau$ is robust to one-shot deviations whenever $\sigma$ is. First consider responder histories where the responders have to react to the proposal $e_{0}$. Since 0 is the lowest possible payoff in the game, it is clear that accepting $e_{0}$ can never be a profitable one-shot deviation. Consider any other history $h$. The action prescribed at $h$ by $\tau$ is the same as that prescribed by $\sigma$. Claim 5.7 says that both strategy profiles lead to the same expected payoffs conditional on $h$, and it also implies that a one-shot deviation, say to action $a$, from $\sigma$ at $h$ leads to the same payoff as a one-shot deviation to $a$ from $\tau$ at $h$. We conclude that if there are no profitable one-shot deviations from $\sigma$, then the same is true for $\tau$. Part [3] of the corollary now follows from Theorem 5.2.

\section{Strategies as finite automata}

In this section we construct a finite automata representation of the strategies used in the proof of Theorem 5.2. A number of different specifications of finite automata have been used in the game-theoretic literature. For a survey of this literature, we refer to Chatterjee and Sabourian (2009). We choose to follow the approach of Chatterjee and Sabourian (2000) as their specification of a machine is particularly convenient for representing strategies in a bargaining game. In 
Chatterjee and Sabourian (2000), a machine consists of a set of states, an initial state, an output function, and a transition function. An important feature of their approach is that the state of the machine does not change during a given round of bargaining. The output function describes the output of the machine as a function of its current state as well as the partial history specifying the actions taken by all the players during the current round of bargaining. A transition from one state to the next only occurs once a given bargaining round has been completed, i.e. once nature has chosen a permutation, a proposal has been made, and has been rejected. The transition function determines the next state of the machine as a function of its current state and the actions of all players in a given round.

Thus, a machine for Player $i \in N$ is a tuple $\left(Q_{i}, q_{i}^{0}, \lambda_{i}, \mu_{i}\right)$, where $Q_{i}$ is the set of states, $q_{i}^{0}$ the initial state, $\lambda_{i}$ the output function, and $\mu_{i}$ the transition function. In accordance with the convention adopted in this paper, we only have to consider two types of partial histories: partial histories specifying only the permutation $\pi$ chosen by nature, and those specifying both the permutation $\pi$ and the proposal $x$. The set of partial histories is $P=\Pi \cup(\Pi \times V)$ and the set of partial histories after which Player $i$ has to move is $P_{i}=\{\pi \in \Pi \mid \pi(1)=i\} \cup\{(\pi, x) \in \Pi \times V \mid \pi(1) \neq i\}$. The output function $\lambda_{i}$ is a function with domain $Q_{i} \times P_{i}$ mapping into the set of actions $A=V \cup$ accept, reject\}, where $\lambda_{i}\left(q_{i}, p_{i}\right) \in V$ if $p_{i} \in \Pi$ and $\lambda_{i}\left(q_{i}, p_{i}\right) \in$ accept, reject $\}$ if $p_{i} \in \Pi \times V$. The outcome of any round of bargaining is specified by $(\pi, x, r) \in \Pi \times V \times N$, where $\pi$ is the permutation chosen by nature, $x$ is the proposal, and $r$ is the player who rejected the proposal. The transition function of the machine is a function $\mu_{i}: Q_{i} \times \Pi \times V \times N \rightarrow Q_{i}$.

Under our representation of the strategies $\sigma_{1}, \ldots, \sigma_{n}$, all players $i \in N$ use the same set of states $Q_{i}$, the same initial state $q_{i}^{0}$, and the same transition function $\mu_{i}$, but possibly different output functions $\lambda_{i}$. We take $Q_{i}=\{\emptyset\} \cup E$ with $\emptyset$ the initial state. Player $i$ 's output function $\lambda_{i}$ is given as follows. Consider some $\pi \in \Pi$. If $\pi(1)=i$, then

$$
\lambda_{i}(q, \pi)= \begin{cases}a, & \text { if } q=\varnothing \\ q, & \text { if } q \in E,\end{cases}
$$

and if $\pi(1) \neq i$, then

$$
\lambda_{i}(q, \pi, x)= \begin{cases}\text { accept, } & \text { if }[q=\emptyset \text { and } x=a], \\ \text { accept, } & \text { if }[q \neq \emptyset \text { and } x=q], \\ \text { accept, } & \text { if }\left[q \neq \emptyset \text { and } x \in E \text { and } x \neq e_{\pi(1)}\right], \\ \text { accept, } & \text { if }\left[x_{i} \geq \delta_{i} \text { and } \pi(n)=i\right], \\ \text { reject, } & \text { otherwise. }\end{cases}
$$

We define the transition function $\mu_{i}$ by

$$
\mu_{i}(\emptyset, \pi, x, r)= \begin{cases}e_{0}, & \text { if } x=a \\ e_{r}, & \text { if } x \neq a\end{cases}
$$

and, for each $q \in E$,

$$
\mu_{i}(q, \pi, x, r)= \begin{cases}e_{0}, & \text { if } x \in E, \\ e_{r}, & \text { if } x \notin E .\end{cases}
$$

Thus each strategy $\sigma_{i}$ is represented by a machine with $n+2$ states. One can similarly use a machine to represent the strategies of Section 3. In fact, one only needs $n+1$ states to do so: the reason is that unlike in our construction, under the Haller-Herrero strategies the vector of zeroes $e_{0}$ is never proposed. This illustrates that counting the length of the strategy's recall and counting the number of states of its finite automaton representation lead to two different measures that capture different aspects of the strategy's complexity. For a more thorough comparison of different approaches to measuring the complexity of a strategy, we refer to Chatterjee and Sabourian (2000, 2009).

\section{Conclusion}

In this paper we have proved a folk theorem for a general class of bargaining games with three or more players. We show that for any vector of feasible payoffs, there is a subgame perfect equilibrium such that the vector of feasible payoffs is proposed and accepted in period 0 . The subgame perfect equilibrium involves pure strategies having one-period recall. The existing literature has shown such a result only for specific examples of bargaining games and relied on strategies having infinite recall.

Our bargaining protocol is sufficiently general to cover many bargaining protocols studied in the literature as special cases. Moreover, it covers cases that have not been studied in the literature so far, where for instance the protocol depends on proposals that have been made in previous rounds. Although our equilibrium strategies have one-period recall, the protocol itself is allowed to depend on aspects of the history that are related to arbitrarily many periods before. Several authors have studied environments where the assumption of bounded recall may severely limit the set of outcomes that can occur in an equilibrium. Our results show that not even one-period recall is sufficient to limit the set of subgame perfect equilibrium payoffs in multilateral bargaining games. 


\section{Appendix A}

Proof of Theorem 3.2. It is clear that the strategy profile $\sigma$ as constructed in Section 3 has the immediate acceptance property. In particular, according to $\sigma$ Player 1 proposes $a$ in period 0 , which is accepted by Players $2, \ldots, n$.

We show that $\sigma$ is a subgame perfect equilibrium provided that $\delta \in\left(\frac{1}{n-1}, 1\right)$.

To do so, it suffices to argue that there are no profitable one-shot deviations from $\sigma$ at any history of the game. Consider a non-terminal history $h$ after which Player $i$ moves. A one-shot deviation by Player $i$ at history $h$ is a strategy $\sigma_{i}^{\prime}$ for Player $i$ that agrees with $\sigma_{i}$ on every non-terminal history except $h$. It is said to be profitable if, conditional on the history $h$ being reached, the payoff to Player $i$ from $\left(\sigma_{i}^{\prime}, \sigma_{-i}\right)$ is higher than that from $\sigma$. As our bargaining game is an infinite horizon discounted multi-stage game with observed actions that is continuous at infinity, the well known one-deviation principle (e.g. Fudenberg and Tirole, 1991) applies.

STEP 1. Consider a proposer history $\left(\pi^{0}, x^{0}, r^{0}, \ldots, \pi^{t-1}, x^{t-1}, r^{t-1}, \pi^{t}\right)$. The strategy profile $\sigma$ leads to a payoff of $\delta^{t} s_{\pi^{t}(1)}\left(x^{0}, \ldots, x^{t-1}\right)$ for Player $\pi^{t}(1)$. Suppose Player $\pi^{t}(1)$ deviates from $\sigma$ and proposes $x^{t} \neq s^{t}\left(x^{0}, \ldots, x^{t-1}\right)$. Since $i^{t+1}\left(x^{t}\right) \neq \pi^{t}(1)$, the proposal $x^{t}$ is rejected by Player $\pi^{t}(2)$ and next period the proposal $s^{t+1}\left(x^{0}, \ldots, x^{t}\right)=e_{i t+1}\left(x^{t}\right)$ will be made and accepted. This leaves Player $\pi^{t}(1)$ with a payoff of 0 .

STEP 2. Consider a responder history $\left(\pi^{0}, x^{0}, r^{0}, \ldots, \pi^{t}, x^{t}\right)$ and a player $j=\pi^{t}(k)$, where $k \in\{2, \ldots, n\}$.

Consider first the case where $x^{t}=s^{t}\left(x^{0}, \ldots, x^{t-1}\right)$. The strategy profile $\sigma$ prescribes acceptance and leads to a payoff of $\delta^{t} x_{j}^{t}$ for Player $j$. If $j$ rejects $x^{t}$, next period the proposal $s^{t+1}\left(x^{0}, \ldots, x^{t}\right)=s^{t}\left(x^{0}, \ldots, x^{t-1}\right)=x^{t}$ will be made and accepted, resulting in a payoff of $\delta^{t+1} x_{j}^{t}$ to Player $j$. Hence rejection is not a profitable deviation.

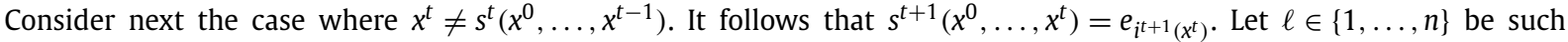
that $\pi^{t}(\ell)=i^{t+1}\left(x^{t}\right)$. We consider three cases depending on whether $k<\ell, k>\ell$, or $k=\ell$.

Case 1: $k<\ell$. According to $\sigma$, Player $j$ should reject, which leads to a payoff of 0 to Player $j$ since the rejection of $x^{t}$ is followed by the proposal $e_{i^{t+1}\left(x^{t}\right)}$, which is accepted. Suppose Player $j$ accepts $x^{t}$. Since $k+1 \leq \ell$, Player $\pi^{t}(k+1)$ will reject $x^{t}$ according to $\sigma$, so also in this case the proposal $e_{i^{t+1}\left(x^{t}\right)}$ is made and accepted in the next period. We conclude that acceptance is not a profitable deviation.

Case 2: $k>\ell$. According to $\sigma$, Player $j$ accepts, which leads to payoff $\delta^{t} x_{j}^{t}$, because the players following $j$ in the response order all accept $x^{t}$ according to $\sigma$. If $j$ rejects $x^{t}$, next period the proposal $e_{i^{t+1}\left(x^{t}\right)}$ will be made and accepted, leaving Player $j$ with payoff 0 . We conclude that rejection is not a profitable deviation.

Case 3: $k=\ell$. In this case $i^{t+1}\left(x^{t}\right)=j$ and the strategy profile $\sigma$ prescribes rejection. It leads to a payoff of $\delta^{t+1}$ to Player $j$ since following the rejection of $x^{t}$ the proposal $e_{i^{t+1}\left(x^{t}\right)}$ will be made and accepted in the next period. Suppose on the other hand that Player $j$ accepts $x^{t}$. Since the players following $j$ in the response order all accept $x^{t}$, this leads to a payoff of $\delta^{t} x_{j}^{t}$. It follows by definition of $i^{t+1}\left(x^{t}\right)$ that $x_{j}^{t} \leq \delta$. We conclude that acceptance is not a profitable deviation.

We have checked that no player has a profitable one-shot deviation from $\sigma$.

\section{References}

Aumann, R.J., 1981. Survey of repeated games. In: Böhm, V., Nachtkamp, H.H. (Eds.), Essays in Game Theory and Mathematical Economics in Honor of Oskar Morgenstern. Bibliografisches Institut, Mannheim, pp. 11-42.

Banks, J., Duggan, J., 2000. A bargaining model of collective choice. Amer. Polit. Sci. Rev. 94, 73-88.

Barlo, M., Carmona, G., Sabourian, H., 2009. Repeated games with one-memory. J. Econ. Theory 144, $312-336$.

Bhaskar, V., Mailath, G.J., Morris, S., 2013. A foundation for Markov equilibria in sequential games with finite social memory. Rev. Econ. Stud. 80, 925-948.

Binmore, K., 1987. Perfect equilibria in bargaining models. In: Binmore, K., Dasgupta, P. (Eds.), The Economics of Bargaining. Basil Blackwell, Oxford, UK, pp. 77-105.

Binmore, K., Osborne, M.J., Rubinstein, A., 1992. Non-cooperative models of bargaining. In: Aumann, R.J., Hart, S. (Eds.), Handbook of Game Theory, vol. 1, pp. 179-225.

Chatterjee, K., Dutta, B., Ray, D., Sengupta, K., 1993. A noncooperative theory of coalitional bargaining. Rev. Econ. Stud. 60, $463-477$.

Chatterjee, K., Sabourian, H., 2000. Multiperson bargaining and strategic complexity. Econometrica 68, 1491-1509.

Chatterjee, K., Sabourian, H., 2009. Game theory and strategic complexity. In: Meyers, R.A. (Ed.), Encyclopaedia of Complexity and Systems Science. Springer Verlag, Berlin, pp. 4098-4114.

Chen, B., 2008. On effective minimax payoffs and unequal discounting. Econ. Letters 100, 105-107.

Cole, H.L., Kocherlakota, N., 2005. Finite memory and imperfect monitoring. Games Econ. Behav. 53, 59-72.

Dutta, P.K., 1995. A folk theorem for stochastic games. J. Econ. Theory 66, 1-32.

Friedman, J., 1971. A non-cooperative equilibrium for supergames. Rev. Econ. Stud. 38, 1-12.

Fudenberg, D., Maskin, E., 1986. The folk theorem in repeated games with discounting or with incomplete information. Econometrica 54, 533-554.

Fudenberg, D., Tirole, J., 1991. Game Theory. MIT Press, Cambridge, Massachusetts.

Fudenberg, D., Yamamoto, Y., 2011. The folk theorem for irreducible stochastic games with imperfect public monitoring. J. Econ. Theory 146, 1664-1683.

Haller, H., 1986. Non-cooperative bargaining of $N \geq 3$ players. Econ. Letters 22, 11-13.

Harsanyi, J.C., 1973. Games with randomly disturbed payoffs: a new rationale for mixed-strategy equilibrium points. Int. J. Game Theory 2, 1-23.

Herings, P.J.J., Meshalkin, A., Predtetchinski, A., 2012. A folk theorem for bargaining games. METEOR Research Memorandum 12/55. Maastricht University, Maastricht, pp. 1-27.

Herings, P.J.J., Predtetchinski, A., 2010. One-dimensional bargaining with Markov recognition probabilities. J. Econ. Theory 145, $189-215$.

Herrero, M.J., 1985. A strategic bargaining approach to market institutions. PhD Thesis. London School of Economics, pp. 1-111.

Kalandrakis, T., 2006. Regularity of pure strategy equilibrium points in a class of bargaining games. Econ. Theory 28, 309-329.

Lehrer, E., Pauzner, A., 1999. Repeated games with differential time preferences. Econometrica 67, 393-412. 
Merlo, A., Wilson, C., 1995. A stochastic model of sequential bargaining with complete information. Econometrica 63, 371-399.

Rubinstein, A., 1979. Equilibrium in supergames with the overtaking criterion. J. Econ. Theory 21, 1-9.

Rubinstein, A., 1982. Perfect equilibrium in a bargaining model. Econometrica 50, 97-109.

Sabourian, H., 1998. Repeated games with M-period bounded memory (pure strategies). J. Math. Econ. 30, 1-35.

Selten, R., 1981. A noncooperative model of characteristic function bargaining. In: Böhm, V., Nachtkamp, H.H. (Eds.), Essays in Game Theory and Mathematical Economics in Honor of Oskar Morgenstern. Bibliografisches Institut, Mannheim, pp. 131-151. 